\title{
Modelo de seguimiento de la prestación económica para cuidados en el entorno familiar ${ }^{1}$
}

\section{Miguel Leturia}

Matia Instituto Gerontológico

<miguel.leturia@matiainstituto.net>

\section{Erkuden Aldaz}

Matia Instituto Gerontológico

\author{
Alberto Uriarte \\ Matia Instituto Gerontológico

\section{Judith Salazar \\ Matia Instituto Gerontológico}

\section{Erlantz Allur \\ Matia Instituto Gerontológico \\ Jon Iribar \\ Diputación Foral de Gipuzkoa}

\section{Zorione Benedicto}

Diputación Foral de Gipuzkoa

Artikulu honek Gipuzkoako Foru Aldundiko Gizarte Politikako Departamentuak Matia Institutu Gerontologikoarekin lankidetzan sustatutako proiektu bat laburtzen du. Azken batean, Familia inguruneko zaintzetarako eta mendekotasuna duten pertsonen zaintzaileen babeserako jarraipen eredua (FIZPE) jasotzea, eta emandako zaintza horien kalitatea eta zaintzaileen bizi-kalitatea bermatzea du helburu. Proiektuak elkarren artean osagarriak diren ondorengo bi lan ildo nagusi hauek uztartzen ditu: batetik, Gipuzkoako 320 familiak parte hartu zuten esperientzia aitzindari bat, eta bestetik, babeserako tresnen diseinua eta aipatutako prestazioa jasotzen den egoeren jarraipen eredua burutzea, guztiak ere, Autonomia Pertsonala Sustatzeari eta Mendekotasun-egoeran dauden Pertsonak Zaintzeari buruzko legearen katalogoan jasoak.

\section{GAKO-HITZAK:}

Bizi-kalitatea, etxebizitzako ingurunea, erreferentziako profesionala, kasuen kudeaketa, prestazio ekonomikoak, zerbitzuak.
Este artículo resume un proyecto impulsado por el Departamento de Política Social de la Diputación Foral de Gipuzkoa, en colaboración con Matia Instituto Gerontológico, con el objetivo de plantear un modelo para el seguimiento de las personas que perciben la prestación económica para cuidados en el entorno familiar (PECEF), y para garantizar en esas situaciones tanto la calidad de los apoyos que recibe la persona dependiente como la calidad de vida de la persona cuidadora. El proyecto integra dos líneas de trabajo paralelas y complementarias: por un lado, una experiencia piloto en la que participaron 320 familias de dos municipios guipuzcoanos; $y$ por otro, el diseño de las herramientas de soporte y la elaboración de un modelo para el seguimiento de las situaciones en las que se percibe la citada prestación, contemplada en el catálogo de la Ley de Promoción de la Autonomía Personal y Atención a las Personas en Situación de Dependencia.

\section{Palabras Clave:}

Calidad de vida, entorno domiciliario, profesional de referencia, gestión de casos, prestaciones económicas, servicios.

\footnotetext{
${ }^{1}$ Este artículo resume dos informes editados por el Departamento de Política Social de la Diputación Foral de Gipuzkoa (2005a y 2005b), disponibles en 〈http://behagi.eus〉.
} 


\section{Introducción}

La bien conocida tendencia de la prestación económica para cuidados en el entorno familiar y apoyo a cuidadores no profesionales (PECEF) a extenderse con relación a otras prestaciones y servicios, a pesar de haber sido considerada excepcional en el texto legislativo, la ha llevado al primer puesto por número de asignaciones del catálogo de servicios y prestaciones. Por tanto, ha adquirido una dimensión imprevista.

A diferencia de otros servicios o prestaciones contemplados en los artículos 14 y 15 de la Ley 39/2006 de Promoción de la Autonomía Personal y Atención a las Personas en Situación de Dependencia, como los centros residenciales, los centros de día, el servicio de ayuda a domicilio, la teleasistencia o incluso otras prestaciones económicas, como las vinculadas a un servicio (PEVS) o la de asistente personal (PEAP), en las que se da una participación de los servicios formales, la incidencia y adecuación de la prestación económica para cuidados en el entorno familiar sólo se puede conocer de la mano de la propia persona dependiente o de sus cuidadores. Es por ello por lo que la Diputación Foral de Gipuzkoa se propuso analizar esta situación y establecer un procedimiento que permitiera intervenir desde antes del acceso a las prestaciones o servicios, para plantear una posterior supervisión de los casos que garantizara la calidad de los cuidados y apoyos (Departamento de Política Social, 2005b). El procedimiento se validó y afinó posteriormente mediante un proyecto piloto desarrollado en las localidades guipuzcoanas de Irun y Arrasate (Departamento de Política Social, 2005a).

El modelo propuesto, por lo tanto, va más allá del propio seguimiento de una situación ya establecida e incorpora el proceso en su conjunto. En efecto, al aplicar el modelo se deberá tener siempre presente que el objetivo de la supervisión es garantizar que la atención se presta efectivamente, con la intensidad suficiente y que la calidad de la atención es adecuada. También, que las personas cuidadoras ejercen su rol en condiciones adecuadas, así como que el entorno familiar y el contexto en el que se realizan los cuidados y la vida ofrecen la suficiente seguridad.

Este artículo resume las dos líneas del proyecto impulsado por el Departamento de Política Social de la Diputación Foral de Gipuzkoa, y realizado en colaboración con Matia Instituto Gerontológico. En primer lugar, se presenta el modelo de seguimiento de las personas que perciben la prestación económica para cuidados en el entorno familiar. Después, se describe brevemente el proyecto piloto llevado a cabo para validar el modelo, proyecto en el que participaron 320 personas beneficiarias de la prestación, y sus familias, residentes en los municipios guipuzcoanos de Irun y Arrasate.

\section{La realidad}

El modelo de seguimiento se considera pertinente en su planteamiento por varias razones y, entre ellas, sería suficiente la que nos muestra la propia realidad. Al cabo de los años, se ha llegado a un número considerable de perceptores de la prestación económica para cuidados en el entorno familiar: a marzo de 2015, en Gipuzkoa son 9.393, lo que supone que la reciben un $45 \%$ de las personas dependientes entre grado III nivel 2 y grado I nivel 2 (las potenciales perceptoras). Como es sabido, a partir del 1 de julio de 2015 las personas con dependencia reconocida posterior a 1 de enero de 2012 en grado I nivel 1 podrán ser, asimismo, perceptoras de las prestaciones económicas (Acuerdo del Consejo Territorial del Sistema para la Autonomía y la Atención a la Dependencia).

En cambio, son 2.415 las personas que reciben de prestación de asistencia personal, un 11,6\%. Este dato es muy significativo y muestra una tendencia de máximo interés, primero, por la propia utilización de esta prestación en los casos de personas mayores dependientes, y segundo, por su evolución creciente en los últimos años. Además del componente cuantitativo, la circunstancia que resulta más determinante para plantear un sistema de seguimiento es el desconocimiento que, en la mayoría de los casos, caracteriza las situaciones de dependencia beneficiarias de la prestación, muchas de ellas con una antigüedad suficiente para que se hayan producido cambios en las variables y circunstancias que configuran estos casos.

Para la elaboración de este proyecto, se han considerado también otras referencias, como la propia regulación de la Diputación Foral de Gipuzkoa sobre las prestaciones económicas (decretos forales 2/2013 y 25/2009), el marco legislativo sobre servicios sociales de la Comunidad Autónoma del País Vasco y el principio de orientación a las personas desde los servicios públicos (Ley 12/2008, art. 7f). Todas ellas refuerzan la oportunidad del modelo de seguimiento, que da asimismo cumplimiento a las conclusiones y orientaciones de trabajos y estudios previos (Siadeco lkerketa Aplikatua, 2013; Benedicto, 2013), impulsados en muchos casos por la Diputación Foral de Gipuzkoa.

Como se recoge en la Tabla 1, en marzo de $\mathbf{2 0 1 5}$, de las 26.623 personas con dictamen de grado de dependencia, y por tanto, beneficiarias de los servicios o prestaciones de la Ley, 9.393 (35,28\%) son perceptoras de la prestación económica para cuidados en el entorno familiar (PECEF); 2.415 (9,07\%), de la prestación económica por asistente personal (PEAP); y 145 (0,54\%), de la prestación económica vinculada al servicio (PEVS). Además, hay 5.801 personas en situación de dependencia con dictamen de grado I, nivel 1 que hasta ahora no podían acceder a las prestaciones económicas. La población total beneficiaria de alguna de estas prestaciones asciende a 11.953 personas, el 44,89\% de las que perciben alguna prestación económica por su situación de dependencia. 
Tabla 1. Prestaciones económicas asignadas, según grado de dependencia. Gipuzkoa, marzo de 2015

\begin{tabular}{|c|c|c|c|c|}
\hline & PECEF & PEAP & PEVS & Perceptores \\
\hline Grado III, nivel 2 & 848 & 424 & 17 & 1.289 \\
\hline Grado III, nivel 1 & 2.011 & 848 & 58 & 2.917 \\
\hline Grado II, nivel 2 & 1.700 & 306 & 21 & 2.027 \\
\hline Grado II, nivel 1 & 3.178 & 541 & 36 & 3.755 \\
\hline Grado I, nivel 2 & 1.656 & 296 & 13 & 1.965 \\
\hline Grado I, nivel 1 & 0 & 0 & 0 & 0 \\
\hline Total & 9.393 & 2.415 & 145 & 11.953 \\
\hline
\end{tabular}

PECEF: prestación económica para el cuidado en el entorno familiar. PEAP: prestación económica de asistencia personal. PEVS: prestación económica vinculada al servicio.

Fuente: Diputación Foral de Gipuzkoa.

Tabla 2. Personas beneficiarias de la prestación económica para cuidados en el entorno familiar y otros servicios complementarios, según puntuación del Baremo de Valoración de la Dependencia. Gipuzkoa, diciembre de 2014

\begin{tabular}{|c|c|c|c|c|c|c|c|c|c|c|}
\hline \multirow[b]{3}{*}{ Puntuación } & \multirow{2}{*}{\multicolumn{2}{|c|}{ BVD }} & \multicolumn{8}{|c|}{ Prestaciones } \\
\hline & & & \multicolumn{2}{|c|}{ SAD } & \multicolumn{2}{|c|}{ CAD } & \multicolumn{2}{|c|}{$S A D+C A D$} & \multicolumn{2}{|c|}{ Sólo PECEF } \\
\hline & $n$ & $\%$ & $n$ & $\%$ & $n$ & $\%$ & $n$ & $\%$ & $n$ & $\%$ \\
\hline $3-2$ & 848 & 8,99 & 85 & 0,90 & 152 & 1,61 & 34 & 0,36 & 577 & 68,04 \\
\hline $3-1$ & 2.022 & 21,43 & 181 & 1,92 & 327 & 3,47 & 60 & 0,64 & 1.454 & 71,91 \\
\hline $2-2$ & 1.714 & 18,17 & 106 & 1,12 & 253 & 2,68 & 46 & 0,49 & 1.309 & 76,37 \\
\hline $2-1$ & 3.189 & 33,80 & 210 & 2,23 & 361 & 3,83 & 60 & 0,64 & 2.558 & 80,21 \\
\hline $1-2$ & 1.662 & 17,62 & 136 & 1,44 & 118 & 1,25 & 14 & 0,15 & 1.394 & 83,87 \\
\hline Total & 9.435 & 100,00 & 718 & 7,61 & 1.211 & 12,84 & 214 & 2,27 & 7.292 & 77,29 \\
\hline
\end{tabular}

BVD: Baremo de Valoración de la Dependencia. SAD: servicio de asistencia domiciliaria. CAD: centros de atención diurna. PECEF: prestación económica para el cuidado en el entorno familiar. Fuente: Diputación Foral de Gipuzkoa.

De los perceptores de prestaciones económicas para cuidados en el entorno familiar, son 7.292 (77,29\%) quienes, a finales de 2014, la están recibiendo sin un servicio formal complementario (Tabla 2).

Un dato destacable, por su relación con la idoneidad y adecuación de esta prestación como respuesta a las situaciones de dependencia, y por su relación con la calidad de la atención y con el rol de la persona cuidadora en el entorno domiciliario, es el de las personas cuidadoras que superan los 75 años (Tabla 3). De los perceptores sin servicios complementarios, en 1.373 casos (18,8\%) se da esta circunstancia, casos todos ellos que merecen una especial consideración.
Tabla 3. Personas cuidadoras de personas beneficiarias de la prestación económica para cuidados en el entorno familiar, por edad. Gipuzkoa, diciembre de 2014

\begin{tabular}{|l|c|}
\cline { 2 - 2 } \multicolumn{1}{l|}{} & $n$ \\
\hline$\leq 64$ años & 4.635 \\
\hline $65-74$ años & 1.284 \\
\hline$\geq 75$ años & 1.373 \\
\hline Total & 7.292 \\
\hline
\end{tabular}

Fuente: Diputación Foral de Gipuzkoa. 
Para acercarnos a la realidad de la prestación como respuesta, más o menos idónea, a las personas en situación de dependencia y a los cuidados y apoyos que requieren, además de los datos cuantitativos, debemos preguntarnos sobre la calidad de vida de las personas en estas circunstancias:

- ¿Colma la prestación las necesidades de atención de las personas con discapacidad, especialmente las de aquéllas con dependencia reconocida de grado III?

- ¿Cómo han evolucionado los casos de asignaciones realizadas hace ya unos años?

- Las personas cuidadoras, algunas de ellas con edad considerable, o que llevan muchos años asumiendo tareas de cuidado, ¿cómo se encuentran?, ¿mantienen las capacidades funcionales y emocionales suficientes?

- ¿Disponemos los servicios sociales de la información suficiente para conocer, no ya la realidad general, sino las diversas realidades que se producen en este ámbito?

\section{Marco regulador}

La prestación económica para cuidados en el entorno familiar y apoyo a cuidadores no profesionales debe ir destinada a la atención de la persona dependiente por cuidadores no profesionales de su entorno familiar y se establece mediante el Decreto Foral 25/2009, de 21 de julio, por el que se regulan las prestaciones económicas de la Ley 39/2006, de 14 de diciembre, de Promoción de la Autonomía Personal y Atención a las Personas en Situación de Dependencia.

La Ley vasca de Servicios Sociales (12/2008) señala expresamente que la atención debe estar personalizada y basarse en una evaluación periódica, la participación de la persona y la asignación de un profesional de referencia, entre otras cuestiones en línea con las consideraciones que el presente modelo de seguimiento también plantea.

\section{La orientación a la persona}

Cualquier relación que establezcan los servicios sociales con la ciudadanía en general, y con las personas en situación de dependencia en particular, debe guiarse por una orientación a la persona que se dirija a la consecución de mejoras en todos los ámbitos de su calidad de vida, partiendo del respeto pleno a su dignidad y derechos, de sus intereses y preferencias, y contando con su participación efectiva (véase, entre otros, Rodríguez Rodríguez, 2013).

Desde este planteamiento, el modelo de supervisión y seguimiento de las situaciones de dependencia perceptoras de la prestación económica para cuidados en el entorno familiar tiene presentes e incorpora estos componentes sustanciales:
- El conocimiento de la persona, un conocimiento global y de sus dimensiones, así como, especialmente, de las características y singularidades de sus intereses, preferencias y deseos.

- Reconocimiento y respeto a la persona: puesta en valor y respeto de la dignidad y singularidad de la persona y, por consiguiente, empatía y comprensión de la perspectiva de la persona.

- Promoción de la autonomía, de modo que se facilite información comprensible para permitir la toma de decisiones, ofreciendo oportunidades y apoyos para realizar elecciones y, en definitiva, para el mantenimiento del control sobre la vida cotidiana.

- Trato personalizado, con flexibilidad en la intervención y seguimiento de los distintos casos para adaptarse a cada persona.

- Consideración del espacio físico y el contexto ambiental tanto como elementos de habitabilidad y promoción de independencia, como de bienestar.

- Relaciones sociales y, sobre todo, familiares, con consideración especial hacia los posibles efectos que la relación de cuidado pueda tener en ellas.

El modelo de seguimiento no pretende implantar un sistema de control/inspección de las prestaciones económicas, sino una necesaria labor de supervisión de las situaciones de dependencia perceptoras de una prestación económica para cuidados en el entorno familiar. Esta supervisión debe basarse en un conocimiento en profundidad y desde antes de la solicitud de la prestación, y en una comunicación permanente, bidireccional y colaborativa entre el servicio público y la familia. Esta relación permanente y personalizada permitirá identificar variaciones, valorar nuevos escenarios, y acordar y articular cambios, para buscar así la mejor adecuación entre necesidades y respuestas.

El objetivo principal del modelo de seguimiento es garantizar que la atención se presta efectivamente con la intensidad suficiente y que la calidad de la atención es adecuada, que las personas cuidadoras ejercen su rol en condiciones adecuadas, y que el entorno familiar y el contexto en el que se realizan los cuidados y la vida ofrecen la suficiente seguridad. Por lo tanto, el alcance va más allá de garantizar la calidad de vida de la persona en situación de dependencia, ya que incorpora a la persona cuidadora y el ejercicio de su rol, así como las posibles consecuencias derivadas del cuidado.

\section{Proceso y fases}

El modelo de supervisión y seguimiento que se plantea para las situaciones de dependencia perceptoras de la PECEF exige entender como un proceso la relación que se establece entre las personas que acceden a los servicios sociales y los profesionales de éstos. Tal como muestra la Figura 1, se trata de un proceso con 
fases diferenciadas, un procedimiento de actuación que permita intervenir, primero, con una asignación de la prestación idónea y, en su caso, con una provisión de apoyos complementarios que aseguren una adecuada atención; y posteriormente, con una supervisión y seguimiento de los casos adecuado y que garantice la calidad de los cuidados y apoyos.

Se analiza, para ello, el proceso en su conjunto, desde la entrada al sistema y su evolución posterior, para llegar a plantear en cada fase del proceso qué acciones/tareas se deben realizar y el tipo de intervención que conviene implantar. Es decir, se examinan la información y la orientación en un inicio, las visitas o contactos de seguimiento -con sus frecuencias correspondientes- en una fase posterior, y la evolución del caso, modificándose la provisión de apoyos o manteniendo la prestación si se concluye que resulta idónea.

Figura 1. Diagrama de flujo del modelo de seguimiento de la prestación económica para cuidados en el entorno familiar

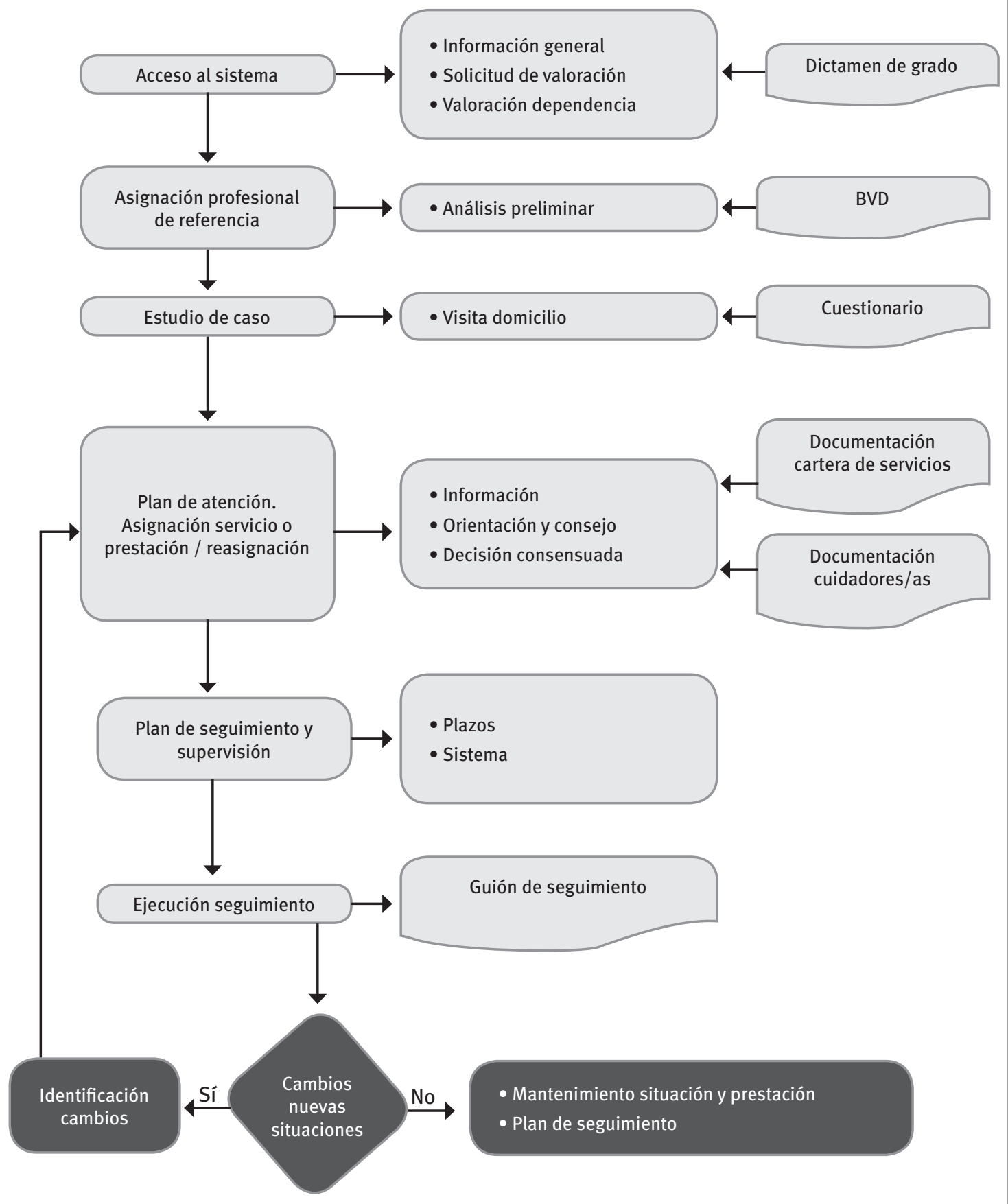

BVD: Baremo de Valoración de la Dependencia.

Fuente: Elaboración propia. 


\subsection{Acceso al sistema}

Se deberá tener presente en todo momento un enfoque claro de 'orientación a la persona'. En este sentido:

- Se establecerá un clima de confianza y de disponibilidad hacia la demanda inicial.

- Se facilitará una información básica sobre los servicios sociales municipales (misión, prestaciones y servicios, funcionamiento), adaptando los contenidos y comunicación a las características apreciadas en la persona demandante en una primera instancia.

- Se explorarán las preferencias de la persona y la familia respecto a las distintas alternativas de servicios o prestaciones para la atención si se cuenta con la información suficiente. En caso contrario, se explicará la cartera de servicios.

- Se informará y asesorará sobre el procedimiento para formalizar la solicitud de valoración de dependencia, se informará sobre la valoración y el posterior dictamen, a partir del cual se pasará a la siguiente fase. Se adelantará que, una vez concluida la valoración, posiblemente se realizará una visita al domicilio para conocer aspectos relevantes relacionados con el interés de seguir residiendo en su entorno de vida.

\subsection{Análisis preliminar}

Para la propuesta del plan de atención y, por consiguiente, del servicio o prestación más adecuado, resulta necesario un análisis inicial de los resultados de la valoración de dependencia (según el baremo establecido por el Real Decreto 174/2011) y conocer, además de su calificación en grado obtenido, el detalle de las valoraciones en las distintas tareas que se contemplan en el Baremo de Valoración de la Dependencia (BVD): el desempeño o no de las actividades, los problemas que se presentan en su ejecución, el nivel de comprensión, el grado de apoyo que recibe de otras personas, información sobre cuestiones de salud relacionadas con el desempeño, la descripción del funcionamiento en la actividad concreta, el empleo de ayudas técnicas, las posibles barreras en el entorno y otras observaciones recogidas. Este análisis debe realizarlo el profesional que se haya identificado como profesional de referencia del caso y que, a partir de este momento, será el que mantenga la relación estable con la persona en situación de dependencia y su familia.

\subsection{Estudio/conocimiento caso}

Realizado el análisis preliminar, que se centra en la persona en situación de dependencia y sus capacidades para el desempeño, así como en la necesidad de apoyos para determinadas tareas y actividades, se debe elaborar un estudio global y en profundidad en tres ámbitos relacionados y condicionados entre sí:
- La persona en situación de dependencia.

- La persona cuidadora en el entorno.

- Las características y condiciones del entorno y contexto.

Esta fase es determinante, y exige acercamiento y proximidad al caso, que se debe realizar en el entorno de vida habitual, en el domicilio. La visita domiciliaria permitirá conocer a los agentes intervinientes en la relación de ayuda que la situación exige, recabar la información necesaria -sistematizada en el cuestionario propuesto- y observar el medio en el que se desarrolla la vida, con sus condicionantes, sean obstaculizadores o facilitadores. La visita domiciliaria facilitará, por tanto, una cuestión determinante en el enfoque que deben tener los servicios y su modelo de atención: hacer partícipe a la persona en el proceso de toma de decisiones. También es una oportunidad para conocer, en parte, su estilo de vida, así como sus preferencias e intereses.

El cuestionario permitirá recoger de manera ordenada la información suficiente para su análisis.

\subsection{Asignación/mantenimiento de la prestación}

El estudio en profundidad del caso y el diagnóstico sobre el grado de adecuación con la prestación permitirán al profesional de referencia diseñar la propuesta de prestación o servicio más ajustada a las necesidades detectadas. Cuando el grado de adecuación sea alto, y además concuerde con los deseos e intereses mostrados por la persona en situación de dependencia y por la familia, la propuesta de prestación será clara y seguirá los trámites establecidos para su asignación definitiva.

En estos casos, se establecerá el plan de seguimiento que el profesional de referencia llevará a cabo posteriormente. En el resto de casos (adecuación media o baja), el profesional de referencia deberá plantearse una intervención en la que:

- Informe sobre cómo valora la situación en su conjunto y las distintas variables que influyen en ella.

- Considere las preferencias de la persona y su familia.

- Valore las opciones de la cartera: las prestaciones, los servicios o ambos, en los casos de compatibilidad.

- Realice una propuesta de plan de atención argumentada en el análisis realizado y que sea comprensible por parte de la persona y su familia.

- Proponga el plan de atención con criterios de flexibilidad y reversibilidad, considerando la satisfacción de la persona y su familia.

- Promueva la toma de decisiones participada y compartida.

- Profundice en la necesidad e identificación de apoyos complementarios. 
- Establezca el plan de seguimiento y supervisión tanto en el tiempo como en el sistema para los casos que acaben siendo perceptores de prestación.

\subsection{Seguimiento y supervisión}

Los casos a los que se ha asignado la prestación económica para cuidados en el entorno familiar deben tener un plan de seguimiento y supervisión, cuyo objetivo principal es garantizar la idoneidad y la alta adecuación entre situación y prestación. Las situaciones de dependencia son dinámicas y cambiantes, evolucionan con ritmos diferenciados, y del mismo modo, las personas que asumen el rol de cuidadoras también cambian.

El plan de seguimiento deberá concretar, sobre todo, dos aspectos:

- La temporalidad, la frecuencia de contacto, que estará en función del caso y de cómo se haya producido la asignación. Habrá casos que necesiten un seguimiento intenso y frecuente, indicador de cierta provisionalidad, y otros en los que no será necesario más que un seguimiento semestral o anual.

- El procedimiento utilizado. Siempre que sea posible, conviene utilizar la visita al domicilio, ya que aporta la información más completa y permite 'aproximarse' a la persona en situación de dependencia, a la persona cuidadora y al entorno de vida. Pueden utilizarse otras procedimientos, como los contactos telefónicos o la entrevista con la persona cuidadora en los servicios sociales de base. Ambos tienen sus limitaciones, pero en situaciones con una adecuación alta pueden garantizar la información necesaria para el seguimiento y supervisión.

\section{Valoración}

\section{1. Áreas y criterios de valoración}

Para que el profesional de referencia pueda concluir, ante cada caso que percibe o solicita percibir la PECEF, el grado de adecuación y, por tanto, la idoneidad y ajuste de ésta, debe analizar los aspectos que expliciten con garantías que:

- La atención se presta con la intensidad suficiente.

- La calidad de la atención es adecuada.

- La persona cuidadora ejerce su rol en condiciones adecuadas.

- El entorno y el contexto en el que se realizan los cuidados y la vida ofrecen la suficiente seguridad. de servicios y prestaciones, la evolución reciente del caso, la sostenibilidad de la situación o el riesgo de claudicación en la provisión de apoyos y cuidados, la organización y gestión de otros apoyos en el entorno cercano del caso, o el contexto en el que se desarrolla la vida cotidiana (entendido como el clima relacional, emotivo y comunicativo).

A la hora de valorar los ámbitos fundamentales y las cuestiones concretas que el cuestionario contempla y que permitirán concluir la adecuación de la PECEF a la situación, se debe tener en cuenta que determinados aspectos que se relacionan con más de uno de los ámbitos de análisis.

\subsubsection{Que la atención se presta con la intensidad suficiente}

Se recogerá la información suficiente, considerando especialmente las cuestiones del cuestionario más relacionadas con la dimensión 'intensidad', como:

- La convivencia.

- La organización y dedicación en distintas horas y días.

- El tiempo de ejercicio del rol de familiar cuidador.

- Otros compromisos dentro o fuera del domicilio.

- Las tareas o apoyos que presentan mayor dificultad o requieren mayor dedicación.

Se deberá concluir si la intensidad se adecua a la situación en grado alto, medio o bajo.

\subsubsection{Que la calidad de la atención es adecuada}

Se recogerá la información suficiente, considerando especialmente las cuestiones del cuestionario más relacionadas con la dimensión 'calidad', como:

- Las consecuencias que la labor y responsabilidad asumida como persona familiar cuidadora pueda tener en diversos ámbitos - relaciones familiares, relaciones y vida social, en el plano emocional y sentimental, físico y de salud-, así como la carga de cuidados.

- Los aspectos positivos del cuidado.

- La intensidad horaria de los cuidados.

- Las dificultades en el cuidado.

- El contexto relacional en el que se producen los cuidados.

- El abordaje de casos con deterioro cognitivo.

Se deberá concluir si la calidad se adecua a la situación en grado alto, medio o bajo. 


\subsubsection{Que la persona cuidadora ejerce su rol en condiciones adecuadas}

Se recogerá la información suficiente, considerando especialmente los aspectos del cuestionario más relacionados con la dimensión "condiciones del ejercicio del rol', como:

- Las consecuencias que la labor y responsabilidad asumida como persona familiar cuidadora pueda tener en diversos ámbitos - relaciones familiares, relaciones y vida social, en el plano emocional y sentimental, físico y de salud-, así como la carga de cuidados.

- Los aspectos positivos del cuidado.

- Qué tipo de cuidados y apoyos afectan más y en qué sentido, en cuanto a dedicación física, en tiempo o en consecuencias emocionales.

- La disponibilidad de ayudas de su entorno más cercano.

- La necesidad de recibir o haber recibido orientaciones, consejos o adiestramiento para la provisión de apoyos y cuidados.

- El interés en disponer de tiempo libre para sí misma por parte de la persona cuidadora.

- La relación con su entorno familiar y social, y la valoración de éstos sobre su rol de cuidar.

- En caso de deterioro cognitivo, los posibles efectos añadidos en cuanto a la complejidad de las tareas de cuidado.

- Las características de la vivienda como facilitadoras u obstaculizadoras para el buen ejercicio del cuidado y para el bienestar de la persona en situación de dependencia.

Se deberá concluir si las condiciones para el ejercicio del rol de cuidados son adecuadas en grado alto, medio o bajo.

\subsubsection{Que el entorno y el contexto en el que se realizan los cuidados y la vida ofrecen la suficiente seguridad}

Se recogerá la información suficiente, considerando especialmente las cuestiones del cuestionario más relacionadas con la dimensión 'entorno y contexto', como:

- Las características de la vivienda desde un punto de vista de accesibilidad, así como de comodidad.

- Los aspectos relativos al contexto en el que se realizan los cuidados tendrán en cuenta la relación con su entorno más cercano, la capacidad para ejercer el rol, la intensidad horaria y la relación entre la persona en situación de dependencia y la persona cuidadora.

- El contexto también está determinado por la relación interpersonal y, en gran medida, por la valoración que muestra el entorno más cercano hacia la persona cuidadora y su labor.
Se deberá concluir si el entorno y contexto se adecua a la situación en grado alto, medio o bajo.

\subsection{Diagnóstico y grado de adecuación de la prestación}

$\mathrm{El} /$ la profesional de referencia debe establecer un diagnóstico de la situación una vez analizada la información recogida por los distintos medios (BVD, cuestionario, visita domiciliaria). No siempre resultará sencillo llegar a ello con plena certeza, al aplicarse un método de análisis en el que, además de información objetiva, se dispondrá de información derivada de la observación y percepción del profesional de referencia, y que puede resultar subjetiva. La competencia profesional en el abordaje y tratamiento de los casos resulta clave en esta función de diagnóstico. Por eso, el/la profesional debe tener siempre presente el marco de las prestaciones y servicios del sistema de atención a la dependencia y la finalidad de cada una de ellas, así como su regulación.

Ello exige también, además de tratar la información y relacionarla en un permanente contraste entre las cuestiones que se tratan, considerar las acciones que puede y conviene aplicar de manera diferenciada y personalizada a cada situación (información, orientación, consejo, apoyos -psicológico o complementarios a la labor de cuidados-) y derivar, en su caso, a otros servicios o prestaciones alternativos.

Siguiendo con el planteamiento del modelo, el/la profesional deberá llegar a establecer para cada caso un grado de adecuación. Puede haber situaciones que exijan un análisis más profundo, por determinadas causas, antes de establecer el diagnóstico. Será necesaria, quizás, una segunda visita o un plazo de provisionalidad hasta determinar las conclusiones definitivas.

\subsubsection{Adecuación alta}

La situación se evalúa como correcta y adecuada. En estos casos, el profesional de referencia establece el plan de seguimiento, señalando y comunicando cuándo volverán a tener un encuentro para revisar la evolución y los posibles cambios. En ocasiones, será suficiente un encuentro semestral o anual, que podrá ir acompañado de llamadas telefónicas tanto del profesional de referencia a la familia como a la inversa.

\subsubsection{Adecuación media}

La situación se considera adecuada, pero se identifican áreas de mejora en uno o varios de los cuatro ámbitos de análisis: la persona en situación de dependencia, la persona cuidadora y la situación de la vivienda. En función de las áreas de mejora, el profesional de referencia procederá a:

- Aportar información, orientación o consejo. 


\begin{tabular}{|c|c|c|c|}
\hline Contexto & $\checkmark$ & $\begin{array}{l}\text { Percepción de la persona cuidadora sobre la capacidad } \\
\text { funcional de la persona dependiente }\end{array}$ & $\checkmark$ \\
\hline Relación de la persona cuidadora con la persona atendida & & Actividades básicas de la vida diaria & \\
\hline Entorno físico & & Actividades instrumentales de la vida diaria & \\
\hline Composición de la unidad convivencial & & Problemas psicosociales & \\
\hline Situación económica & & Deterioro cognitivo & \\
\hline Calidad de las relaciones familiares & & Problemas conductuales & \\
\hline Tiempo que la persona lleva ejerciendo los cuidados & & Necesidades sanitarias & \\
\hline \multicolumn{4}{|l|}{ Situación laboral } \\
\hline Valores y preferencias de la persona cuidadora & $\checkmark$ & Bienestar de la persona cuidadora & $\checkmark$ \\
\hline Deseo o voluntad de asumir la atención & & Percepción de su estado de salud & \\
\hline $\begin{array}{l}\text { Deseo o voluntad de la persona atendida de recibir } \\
\text { atención }\end{array}$ & & Diagnósticos o sintomatología & \\
\hline Sentimiento de obligación de cuidar & & Depresión o ansiedad & \\
\hline Preferencias para organizar la atención & & Grado de satisfacción o calidad de vida & \\
\hline Consecuencias de la atención informal & $\checkmark$ & Habilidades/conocimiento para prestar la atención & $\boldsymbol{V}$ \\
\hline Aislamiento social & & $\begin{array}{l}\text { Capacidad y habilidades, prestar apoyo en las actividades } \\
\text { de la vida diaria (AVD) }\end{array}$ & \\
\hline Excesiva carga emocional & & $\begin{array}{l}\text { Conocimiento y habilidades para prestar apoyos de } \\
\text { atención sanitaria }\end{array}$ & \\
\hline Excesivo esfuerzo físico & & Habilidades para afrontar trastornos de comportamiento & \\
\hline Excesiva carga económica & & & \\
\hline Estrés en relaciones familiares & & & \\
\hline Desarrollo de nuevas habilidades & & & \\
\hline Satisfacción derivada & & & \\
\hline Mejora de las relaciones familiares & & & \\
\hline Recursos de apoyo disponibles & $\checkmark$ & Otras observaciones & $\checkmark$ \\
\hline $\begin{array}{l}\text { Disponibilidad de apoyos informales de otros familiares/ } \\
\text { allegados }\end{array}$ & & & \\
\hline $\begin{array}{l}\text { Disponibilidad de servicios formales de respiro, ayuda } \\
\text { domicilio o atención diurna, nocturna, e interés en ellos }\end{array}$ & & & \\
\hline Apoyos & & & \\
\hline
\end{tabular}

Fuente: Elaboración propia a partir de SIIS Centro de Documentación y Estudios (2010: 114-115).

- Solicitar algún apoyo complementario, establecer un servicio compatible con la prestación económica.

- Solicitar alguna ayuda técnica.

- Gestionar algún apoyo al cuidador/a principal, bien de respiro (sustitución por un profesional determinadas horas a la semana, estancias temporales) bien en forma de asesoramiento en el propio domicilio (determinando los aspectos concretos de mejora).

- Informar del programa Sendian.

- Valorar la oportunidad de acompañamientos a través de voluntariado, tanto en el domicilio como en el exterior de la vivienda.

\subsubsection{Adecuación baja}

La situación se juzga insostenible por diversos motivos, habitualmente relacionados con la evolución de la dependencia, con la capacidad del familiar cuidador o con la situación vivienda. En estos casos, se plantean las siguientes opciones:

- Cambiar la prestación económica para cuidados en el entorno familiar (PECEF) por la prestación económica de asistencia personal (PEAP).

- Proponer ayudas técnicas.

- Sugerir reformas asequibles y ayudas existentes para mejorar la vivienda. 
- Informar, orientar y aconsejar sobre apoyos y programas para cuidadores informales.

- Informar, orientar y aconsejar sobre otros servicios de cartera.

- Revalorar la situación de dependencia.

Tal como se reseña en el artículo 8d de la Ley 12/2008, de 5 de diciembre, de Servicios Sociales del País Vasco, se "asignará a cada persona o familia un profesional o una profesional de referencia en el Sistema Vasco de Servicios Sociales, al objeto de garantizar la coherencia de los itinerarios de atención y la coordinación de las intervenciones". En el desarrollo de las funciones de esta figura, tiene, por tanto, un perfecto encaje el planteamiento de trabajo que se establece en este modelo de supervisión y seguimiento de las situaciones en las que se percibe la prestación económica para cuidados en el entorno familiar.

\subsection{Funciones del profesional de referencia}

La necesaria coherencia de los itinerarios presupone -y así es, de hecho- que las situaciones de dependencia son dinámicas y, en consecuencia, evolucionan y cambian. Estos cambios suelen exigir el incremento o la variación de los apoyos requeridos, tanto en complejidad como en intensidad horaria, por parte del entorno más cercano, el familiar. De ahí que sea necesario su seguimiento, su análisis, para identificar cambios y valorar el grado en que la prestación asignada y su finalidad se adecuan a las nuevas circunstancias detectadas.

Sin ser, en todo su alcance, una metodología de gestión de casos, al no pretender coordinar un amplio abanico de servicios posibles y de profesionales relacionados a lo largo de un itinerario integral de intervención, el procedimiento que se plantea sí comparte el fundamento de esta metodología. El modelo propuesto supone un 'acompañamiento' a lo largo de un itinerario, aunque éste gira en torno a una situación concreta, que es la de los perceptores de la PECEF.

La acción del profesional de referencia corresponde a una intervención que proporciona información experta y cualificada, facilita asesoramiento sólido, y monitoriza el caso individual, haciendo partícipe en ese proceso a la persona beneficiaria. Este profesional acompaña mediante dicho planteamiento de supervisión y seguimiento a la persona y a su familia, con el fin de garantizar la calidad de la atención, considerando tanto a la persona en situación de dependencia como a la persona cuidadora en su ejercicio de atender, en su rol de cuidadora, y además a lo largo del tiempo.

En síntesis, la acción del profesional de referencia se centra en:

- La persona, sus necesidades, intereses, preferencias y deseos, cultura, recursos propios y de su entorno cercano; en vez de priorizar los intereses institucionales de los servicios.

- Tener presente la promoción de la autonomía de la persona como guía de la intervención.

- La intervención del profesional de referencia se realiza previo estudio, diagnóstico, valoración y evaluación de las situaciones y necesidades actuales de la persona. Corresponde a una actuación profesional competente, que permite un conocimiento global y en profundidad de cada caso.

- La priorización de objetivos (mantenimiento en el domicilio, identificación de apoyos necesarios en intensidad y calidad, condiciones para el ejercicio de rol de cuidado adecuado, y en un entorno y contexto facilitadores, definidos temporalmente en función de su realidad actual y posible evolución, y consensuados con la persona de acuerdo con la valoración de sus necesidades (actuales), sus capacidades (personales, culturales, de recursos, relacionales) y de sus intereses y decisiones.

- Un enfoque de proximidad, porque 'se acerca' al espacio personal de la persona y la familia, y trabaja conjuntamente con ellas.

- La elaboración participada de propuestas. Facilita la determinación o decisiones consensuadas mediante un proceso profesional comunicacional.

- La evaluación del proceso concretado en un plan de supervisión y seguimiento.

\section{La experiencia piloto}

En este último apartado, se describe de forma resumida el planteamiento y principales resultados del proyecto piloto desarrollado con 320 familias beneficiarias de la prestación económica para cuidados en el entorno familiar en los municipios guipuzcoanos de Irun y Arrasate (Departamento de Política Social, 2015b). Mediante esta intervención, se ha podido afinar y validar el modelo de seguimiento de dicha prestación expuesto en la primera parte del artículo.

\subsection{Identificación de la muestra}

Matia Instituto Gerontológico, junto con la Diputación Foral de Gipuzkoa, estableció en primer lugar los criterios empleados para identificar la muestra objeto de estudio, que fueron los siguientes:

- Expedientes de PECEF iniciados entre 2008 y 2011.

- Situaciones de dependencia de grados II y III.

- No disposición de otro tipo de servicios complementarios.

Una vez identificadas todas las personas que cumplían los criterios de acceso a la muestra del estudio, se dividieron por grupos de edad y se entrevistó al $65 \%$ de las personas de cada grupo, elegidas alea- 
Tabla 4. Muestra inicial según criterios de perfil

\begin{tabular}{|c|c|c|c|c|c|c|c|}
\hline \multirow{2}{*}{$\begin{array}{l}\text { Año de inicio del } \\
\text { expediente }\end{array}$} & \multicolumn{3}{|c|}{ Irun } & \multicolumn{3}{|c|}{ Arrasate } & \multirow{2}{*}{ Total } \\
\hline & BVD grado III & BVD grado II & Total & BVD grado III & BVD grado II & Total & \\
\hline 2008 & 32 & 30 & 62 & 23 & 12 & 35 & 97 \\
\hline 2009 & 42 & 63 & 105 & 40 & 11 & 51 & 156 \\
\hline 2010 & 18 & 49 & 67 & 7 & 19 & 26 & 93 \\
\hline 2011 & 46 & 24 & 70 & 6 & 24 & 30 & 100 \\
\hline Total & 138 & 166 & 304 & 76 & 66 & 142 & 446 \\
\hline
\end{tabular}

BVD: Baremo de Valoración de la Dependencia.

Fuente: Elaboración propia.

toriamente. Además, se seleccionaron, por considerarse un grupo prioritario, todos aquellos expedientes en los que figurara una persona cuidadora mayor de 75 años, independientemente de la edad de la persona en situación de dependencia. El número total de entrevistas realizadas fue de 320 , cifra que se considera más que suficiente para extraer conclusiones sobre las condiciones de vida de las personas en situación de dependencia en ambos municipios, y para establecer un modelo de seguimiento acorde con los resultados obtenidos en esta experiencia.

Una vez organizada la agenda de citas, las trabajadoras sociales contaron con la presencia de los coordinadores de Matia Instituto Gerontológico en aproximadamente la mitad de los encuentros. Las visitas domiciliarias siguieron siempre un mismo procedimiento, que tenía por objetivo que las familias aceptaran la visita y crear un clima apropiado para ella. Una vez en el domicilio, se les recordaba el motivo de la visita (conocer la situación actual e identificar cambios que pudieran incidir en la valoración de dependencia) y se recogía una primera impresión de la persona cuidadora sobre las circunstancias en que se desarrollaba la relación de cuidados. Después, se les explicaba que la entrevista se dividía en tres partes. En un primer lugar, se solicitaba que mostraran la vivienda, para observar su grado de adecuación a las necesidades de la persona en situación de dependencia y detectar eventuales dificultades de accesibilidad. En segundo lugar, se informaba a la familia de que gran parte de la entrevista se realizaba con la persona cuidadora, para poder comprender cuál era su situación y de qué manera desarrollaba su rol de cuidadora principal. En la parte final de la visita, se entrevistaba a la persona en situación de dependencia. Las entrevistas con la persona cuidadora y con la persona dependiente se realizaban por separado si se entendía posible y adecuado.

\subsection{Cuestionario}

El principal objetivo de la supervisión realizada en el domicilio es garantizar que la atención se presta efectivamente con la intensidad suficiente y que la calidad de la atención es adecuada. También, que las personas cuidadoras ejercen su rol en condiciones adecuadas, así como que el entorno familiar y el contexto en el que se realizan los cuidados y discurre la vida ofrecen la suficiente seguridad. Para ello, el cuestionario se dividió en cinco bloques:

- Relación con los servicios sociales municipales: se pretende conocer si existe relación entre la persona dependiente y los servicios sociales municipales.

- Domicilio y situación de la vivienda: se trata de recoger información sobre la vivienda en la que reside la persona dependiente, para saber si existen barreras que obstaculicen la labor de cuidado y la calidad de vida de esa persona. Se considera además que la observación del domicilio ofrece mucha información complementaria que no se puede obtener de otra manera (sobre situación familiar, higiene, relaciones familiares) y, por tanto, permite detectar situaciones que permitan valorar el grado de adecuación de la atención prestada.

- Persona cuidadora. Se abordan cuatro cuestiones:

- Información básica: grado de parentesco entre persona dependiente y cuidadora, existencia de convivencia, relación entre ambas personas.

- Organización de los cuidados: tiempo que se dedica, existencia de apoyos familiares o profesionales, tiempo que lleva atendiendo a la persona dependiente y evolución de sus necesidades.

- Consecuencias del cuidado en la persona cuidadora: situación emocional, influencia de los cuidados en la vida social y familiar, y en el estado de salud. Se trata de detectar asimismo la existencia de sobrecarga (Zarit, Reever y Bach-Peterson, 1980).

- Dificultades y servicios existentes: este subapartado pretende recoger las dificultades que encuentra la persona cuidadora en su tarea de cuidado, con objeto de realizar orientaciones y proponer servicios que pudieran ayudarle en su labor.

- Persona dependiente: el objetivo de este bloque es conocer el grado de satisfacción de los cuidados que recibe, las relaciones familiares y los apo- 
yos de los que dispone la persona en situación de dependencia. Asimismo, se pretende conocer el grado de satisfacción con la atención sanitaria recibida y facilitar información de recursos, conociendo de antemano su opinión sobre ellos.

- Profesional que realiza la entrevista: el cuestionario cuenta con ítems que no se preguntan a las familias, sino que se cumplimentan tras la observación del profesional de referencia. Así, se pretende realizar una comparación entre el Baremo de Valoración de la Dependencia (BVD) de origen y la situación que se ha observado, detectar posibles indicadores de trato no adecuado, $y$ determinar el grado de adecuación de la situación observada y realizar alguna orientación al respecto, en caso de ver necesidad.

\subsection{Algunas conclusiones}

De las entrevistas realizadas en el domicilio, se extrae que la atención se presta efectivamente con la intensidad suficiente. Además, y salvo casos puntuales, la calidad de la atención es adecuada. Apenas se han identificado indicadores de trato inadecuado y, en general, el estilo relacional y la comunicación con la persona dependiente son apropiados. Asimismo, en su conjunto las personas cuidadoras reúnen las condiciones mentales, físicas y psicológicas para hacer frente con calidad al cuidado, aunque se hayan detectado casos en los que dichas condiciones se ponen en cuestión.

De lo anterior se desprende nuevamente la necesidad de realizar un seguimiento continuado de cada caso, teniendo presente que la evolución de cada uno es desigual en el tiempo y heterogénea en su contexto. También se confirma la necesidad de prestar especial atención a los perfiles de mayor vulnerabilidad, como las personas cuidadoras de avanzada edad.

La práctica totalidad de las personas en situación de dependencia se sienten satisfechas con la atención que reciben tanto por parte de la cuidadora principal como por parte de los servicios sanitarios, así como con las relaciones que mantienen con su entorno más próximo. Sin embargo, es de destacar que casi una cuarta parte de las personas entrevistadas refieren no recibir visitas y es significativo el número de quienes no han salido a la calle en el último mes. Ambos aspectos deben tomarse en consideración, dado que podrían conllevar el aislamiento de las personas en situación de dependencia.

Aparte de la calidad de la atención, se puede concluir que, en general, las condiciones en las que las personas cuidadoras ejercen su rol son también adecuadas, si bien existen áreas a las que prestar atención para garantizar esta situación a lo largo de los años.
Como se desprende de los resultados, las personas cuidadoras se sienten valoradas y reconocidas en su rol de cuidar, y no presentan sobrecarga derivada del cuidado, pese a que su dedicación es intensa y continuada a lo largo de los años. No obstante, parece que han asimilado su rol y se han adaptado a él, no sin presentar consecuencias tanto positivas como negativas por ello. Una amplia mayoría se siente satisfecha de lo que hace por la persona a la que ayuda. Los efectos negativos que el cuidar desencadena en sus vidas son más evidentes en tres áreas: las relaciones sociales, la salud física y la salud emocional.

Así, dada su prevalencia y su importante repercusión en la calidad de la atención, se considera necesario abordar estas cuestiones con las personas cuidadoras y tenerlas muy en consideración a la hora de estudiar cada caso, al realizar una orientación y en el seguimiento. En este sentido, es de destacar la importancia de la información y la orientación profesional. En línea con la necesidad de orientación por parte de profesionales, cabe señalar que la mayoría de los casos visitados han agradecido el hecho de que se haya acudido al domicilio con el fin de conocer la situación, interesarse por sus condiciones de vida y labor cuidadora, y orientar en caso necesario. Este tipo de acción supone un reconocimiento de la labor de cuidado que realizan las familias y cierto refuerzo positivo, que, si se generalizara, se convertiría en un apoyo complementario tanto para la persona cuidadora como para aquélla en situación de dependencia.

A lo largo del estudio, se ha constatado que la vivienda y sus características suponen, para más de una tercio de las familias, un inconveniente tanto para el desarrollo de la labor de cuidado como para la calidad de vida de las persona en situación de dependencia. El baño y el dormitorio de la persona dependiente son las estancias que mayores dificultades presentan. Con respecto al edificio, pese a que la mayoría dispone de ascensor, destaca la elevada proporción (un tercio) que tienen escaleras intermedias que obstaculizan el acceso, sin que exista sistema alternativo para compensar dicha dificultad.

Otro aspecto importante del cuidado es el contexto relacional de la familia. A este respecto, cabe señalar que, en su mayoría, el contexto en el que se desarrolla la tarea de cuidado es el idóneo. La relación entre persona cuidadora y persona dependiente es buena, y el trato, adecuado. Ambas manifiestan asimismo su satisfacción con las relaciones que mantienen con el resto de familia y con el entorno social, si bien las consecuencias del cuidado en el ámbito familiar y social son pronunciadas. No obstante, ello parece afectar a la intensidad de la relación (tiempo para estar con otros familiares o con amigos) en lugar de a su calidad. 
BENEDICTO, Z. (2013): “La prestación económica de asistencia personal en el territorio histórico de Gipuzkoa”, Zerbitzuan, nํㅜ 54, págs. 67-84 [〈https://dx.doi.org/10.5569/1134-7147.54.04'].

DEPARTAMENTO DE POLÍTICA SOCIAL (2015a): Experiencia piloto: estudio de la situación de las personas en situación de dependencia y de sus cuidadores en los municipios de Irún y Arrasate. Informe, Donostia-San Sebastián, Diputación Foral de Gipuzkoa [«http://behagi. eus/files/informes/experiencia-piloto-estudiode-la-situacin-de-las-personas-en-situacin-dedependencia.pdf $>$.

- (2015b): Modelo de seguimiento de la Prestación Económica para Cuidados en el entorno y apoyo a las Personas Cuidadoras de Personas en situación de Dependencia, Donostia-San Sebastián, Diputación Foral de Gipuzkoa [rhttp://behagi.eus/files/informes/modelo-deseguimiento-de-la-prestacin-econmica-paracuidados.pdfı].

ESPAÑA (2012): "Resolución de 13 de julio de 2012, de la Secretaría de Estado de Servicios Sociales e Igualdad, por la que se publica el Acuerdo del Consejo Territorial del Sistema para la Autonomía y Atención a la Dependencia para la Mejora del Sistema para la Autonomía y Atención a la Dependencia", Boletín Oficial del Estado, no 185 , 3-8-12, págs. 55.65755.674 ['https://www.boe.es/buscar/doc. php?id=BOE-A-2012-10468>].

- (2011): “Real Decreto 174/2011, de 11 de febrero, por el que se aprueba el Baremo de Valoración de la Situación de Dependencia Establecido por la Ley 39/2006, de 14 de diciembre, de Promoción de la Autonomía Personal y Atención a las Personas en Situación de Dependencia”,
Boletín Oficial del Estado, no 42, 18-2-2011, págs. 18.567-18.691 [<https://www.boe.es/ buscar/doc.php?id=BOE-A-2011-3174'].

- (2006): “Ley 39/2006, de 14 de diciembre, de Promoción de la Autonomía Personal y Atención a las Personas en Situación de Dependencia", Boletín Oficial del Estado, nํ-299, 15-12-06, págs. 44.142-44.156 [rhttps://www.boe.es/boe/ dias/2006/12/15/pdfs/A44142-44156.pdf〉].

GIPUZKOA (2013): “Decreto Foral 2/2013, de 22 de enero, de Modificación del Decreto Foral 25/2009, de 21 de julio, por el que se Regulan las Prestaciones Económicas de la Ley 39/2006, de 14 de diciembre, de Promoción de la Autonomía Personal y Atención a las Personas en Situación de Dependencia", Boletín Oficial de Gipuzkoa, no 17, 25-1-2013, págs. 2-4 [rhttps://ssl4. gipuzkoa.net/castell/bog/2013/01/25/ c1300710.pdf〉].

- (2009): “Decreto Foral 25/2009, de 21 de julio, por el que se Regulan las Prestaciones Económicas de la Ley 39/2006, de 14 de diciembre, de Promoción de la Autonomía Personal y Atención a las Personas en Situación de Dependencia", Boletín Oficial de Gipuzkoa, no 144, 4-8-2009, pág. 2 [<https://ssl4.gipuzkoa.net/castell/ bog/2009/08/04/(c0909608.pdf)].

PAÍS VASCO (2008): “Ley 12/2008, de 5 de diciembre, de Servicios Sociales", Boletín Oficial del País Vasco, no-246, 24-12-2008, págs. 31.84031.924 [khttp://www.euskadi.net/bopv2/ datos/2008/12/0807143a.pdf>].

RODRÍGUEZ RODRÍGUEZ, P. (2013): La atención integral y centrada en la persona, serie Papeles de la Fundación, no 1, Fundación Pilares para la Autonomía Personal [<http://www. fundacionpilares.org/docs/AICPweb.pdfs]. 
SIADECO IKERKETA APLIKATUA (2013): "Las prestaciones económicas de dependencia en Gipuzkoa: concurrencia, impacto y supervisión", Zerbitzuan, no 54 , págs. 47-67 ['https://dx.doi. org/10.5569/1134-7147.54.03'].

SIIS CENTRO DE DOCUMENTACIÓN Y ESTUDIOS SIIS (2010): Prestaciones económicas y otras fórmulas de asignación económica individual en los sistemas de atención a la dependencia. Panorama comparativo internacional, Donostia-San Sebastián, Diputación Foral de Gipuzkoa [<http://behagi.eus/files/ informes/prestaciones-economicas-otras- formulas-asignacion-economica-dependenciainternacional.pdf $\rangle$.

ZAHARTZAROA-ASOCIACIÓN VASCA DE GERIATRÍA Y GERONTOLOGÍA (2014): Instrumento de detección de señales de alerta de malos tratos físicos y económicos a personas mayores en la CAPV, Vitoria-Gasteiz, Eusko JaurlaritzaGobierno Vasco.

ZARIT, S. H.; REEVER, K. E.; y BACH-PETERSON, J. (1980): "Relatives of the impaired elderly: Correlates of feelings of burden", The Gerontologist, vol. 20, no 6, págs. 649-655 [khttp://dx.doi. org/10.1093/geront/20.6.649']. 